\title{
Simulation of Patellofemoral Joint Reconstruction Surgery on Patient-Specific Models
}

\author{
Zohara A. Cohen and Gerard A. Ateshian \\ Department of Mechanical Engineering, Columbia University \\ Orthopaedic Research Laboratory, Department of Orthopaedic Surgery, \\ Columbia-Presbyterian Medical Center \\ 630 West 168th St, BB1412, New York, NY 10032
}

\begin{abstract}
The outcome of patellofemoral joint reconstructive surgery has been found to be variable. This study presents patient-specific models of the patellofemoral joints of 13 patients diagnosed with osteoarthritis. The model is a mathematical model using quasi-static equilibrium analysis and the model inputs are patient data is acquired from a combination of two series of magnetic resonance images. Four tibial tuberosity transfer surgeries are simulated for each patient, namely: $15 \mathrm{~mm}$ anterior shift, $20 \mathrm{~mm}$ anterior shift, $8 \mathrm{~mm}$ anterior along with $8 \mathrm{~mm}$ medial shift, and $15 \mathrm{~mm}$ anterior along with $8 \mathrm{~mm}$ medial shift. The simulated surgeries produced a statistically significant decrease in mean contact stress relative to pre-surgical conditions $(\mathrm{p}=0.004)$, though no statistical difference was found between the different procedures. No one procedure consistently demonstrated the best outcome for all patients. In fact, certain surgical procedures increased peak stresses in some subjects while decreasing it in others. With the demonstrated variability of outcome, the utility of the model presented for surgical planning becomes apparent.
\end{abstract}

\section{Introduction}

\subsection{Tuberosity Transfer: Variable Surgical Outcome}

Clinically, patellofemoral joint (PFJ) reconstruction surgeries have met with inconsistent success. In particular, surgeries that aim to restore a lower stress level in the painful PFJ by shifting the tibial tuberosity have been reported in the clinical literature to yield variable outcomes. In 1976 Maquet introduced his surgical correction in which the tibial tuberosity, the bone protrusion where the patellar ligament inserts on the tibia, is transferred $20-25 \mathrm{~mm}$ anteriorly to increase the moment arm of the patellar ligament and thereby decrease the amount of quadriceps force needed to effect extension of the tibia [18]. In a modification to the Maquet procedure, Fulkerson et al. recommended a procedure in which the tibial tuberosity is cut along an antero-medial plane and then translated along that plane [12]. Such a procedure creates a medial and anterior displacement of the patellar ligament insertion without requiring a bone graft. These authors performed a cadaver study in which they combined $8 \mathrm{~mm}$ of medialization with both 8 and $15 \mathrm{~mm}$ anteriorization and found the procedure to decrease the overall contact stress, while at the same time achieving a better balance of forces between the medial and lateral aspects of the trochlear groove.

While some clinical studies of tuberosity transfer procedures show good to excellent results in as many as $80 \%$ (10-20 years follow up) [21] or $85 \%$ (1-4 years 
follow up) [19] of cases, others report a success rate of only 54\% (6 years follow up) [13]. In vitro cadaver studies have also shown inconsistent results with respect to decrease in contact stress $[10,11,17]$. Generally, cadaver studies have revealed that the optimal relief of stress occurs with smaller amounts of anteriorization than Maquet had originally suggested: $10 \mathrm{~mm}$ according to one study by Ferrandez, et al., [11] and $12.5 \mathrm{~mm}$ according to Ferguson, et al., [10]. Others reported that $25 \mathrm{~mm}$ of anteriorization leads to an inconsistent decrease in stress [17]. Three-dimensional (3D) computer models generated from digitized cadaver data have also been used to assess the effectiveness of tibial tuberosity transfer $[4,6,14,20]$.

A limitation of these in vitro studies is their use of cadaver data that most likely derive from cadavers with no pathology. Nevertheless, the variable mechanical outcome observed in the cadaver studies, which are free of biological complications, indicates that the frequent failure of the clinical procedure may be due to a flaw in the mechanical basis for the procedure. Given, however, that many cases do achieve success, the mechanical benefit of the procedure may vary according to the geometry of the particular knee. With the advent of medical imaging, 3D joint models can be created from in vivo patient data.

\subsection{Objective of Current Study}

The long-term objective of this study is to determine whether computer simulation of various tuberosity transfer procedures performed on patient-specific multibody model of the PFJ can provide guidance as to the preferred procedure for that patient. In the current study we created patient-specific multibody joint models by acquiring articular geometry, tendon insertion points and kinematic information from two sets of magnetic resonance images. The patients in the study have all been diagnosed with patellofemoral joint osteoarthritis (OA) with lateral patellar tracking and, thus, are candidates for tibial tuberosity transfer procedures. In light of the prior work regarding the optimal amount of tuberosity anteriorization, we simulated two Maquet-style tuberosity transfers, $15 \mathrm{~mm}$ and $20 \mathrm{~mm}$ anterior shift (to be referred to in this paper as M15 and M20). We also simulated two Fulkerson-style procedures, $8 \mathrm{~mm}$ medial $-8 \mathrm{~mm}$ anterior, and $15 \mathrm{~mm}$ anterior $-8 \mathrm{~mm}$ medial (F8-8 and F15-8), yielding a total of four procedures for each patient. Following each surgical simulation the state of stress in the joint was compared to the pre-surgery configuration. Based on the clinical literature, the hypothesis of the study was that the surgical outcome, assessed from certain biomechanical variables, is patient specific and that no single procedure produces superior results across the board. The long-term corollary hypothesis is that the optimum procedure for a given patient can be discerned, thereby improving the clinical outcome of patellofemoral joint reconstruction.

\section{Methods}

\subsection{Acquisition of Patient Data}

Thirteen patients were selected for the study because of clinical diagnosis of PFJ OA with lateral subluxation (a condition where the patella tracks on the lateral aspect of the femoral trochlea and is usually associated with dysplasia of the vastus medialis obliquus) and no prior PFJ reconstruction surgery. The surface topographies of the subchondral bone and articulating surface of the cartilage were generated by segmenting magnetic resonance images (MRI) and fitting b-spline surfaces to the 
segmented data. The first imaging sequence highlighted articular cartilage and was a fat suppressed spoiled gradient recalled sequence (SPGR) acquired with an extremity coil, with the knee in full extension, at a resolution of $0.55 \times 0.55 \times 1.5 \mathrm{~mm}^{3}$, and requiring 9 minutes (Fig 1). The images were segmented with a semi-automated snake algorithm that has been shown to have an accuracy of $0.23 \mathrm{~mm}$ for topography measurement and $0.37 \mathrm{~mm}$ for thickness measurement [7].

A second series was acquired in the full-body coil to determine the kinematic position of the knee bones in a more functional flexed position. These images were T1 weighted with a resolution of $0.98 \times 0.98 \times 4.0 \mathrm{~mm}^{3}$ and required 4.5 minutes (Fig 1). The bone contours from the extended images were registered with the flexed images to determine the correct kinematic position in which to place the highresolution bone and cartilage surfaces [8].
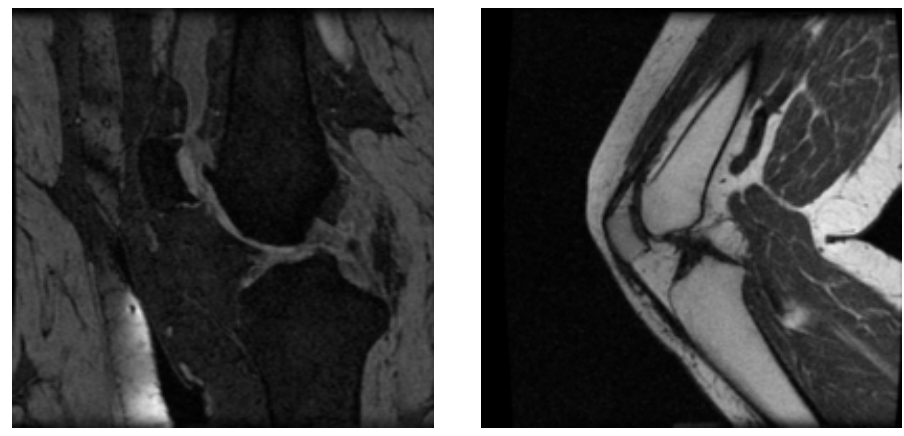

Figure 1:

Left: highresolution image; knee in extended position

Right: large field of view image; knee in flexed position

\subsection{D Multibody Modeling of the Patellofemoral Joint}

Patellofemoral joint kinematics were simulated using a mathematical multibody model which employs quasi-static equilibrium analysis to predict the position of interacting bodies [16]. The articular layers in the model are represented by mathematical surfaces and the contact stress between them approximated by a function of the local strain. In regions of thinner cartilage, the same overlap distance produces a larger strain and, thus, an apparent stiffening of the cartilage [5]. (To read more about the algorithm for calculating overlap distance, see [3].) Furthermore, the bone is assigned a higher compressive modulus than the cartilage so that contact with the bone generates accordingly higher stresses. Ligaments are modeled as passive line elements that exert forces dependent on their length. Prescribed muscle forces are applied via tendon elements whose direction is guided by their points of origin on one bone and insertion points on other bones. For the PFJ model, the quadriceps muscles were loaded in 3 groups: vastus lateralis (VL); rectus femoris (RF) + vastus intermedius (VI) + vastus medialis longus (VML); and vastus medialis obliquus (VMO). The muscle groups were loaded in the ratio 2:3:1 following the findings in cadavers for relative physiological cross-sectional areas of the quadriceps muscles by Ahmed [1] and Farahmand [9].

\subsection{Simulation of Surgery}

To simulate the weakened state of the VMO for patients with lateral tracking, the portion of the total quadriceps force carried by the VMO was cut in half relative to the proportions reported in our prior cadaver calibration study [16] yielding a ratio of 4:6:1 for VL:RF+VI+VML:VMO. 

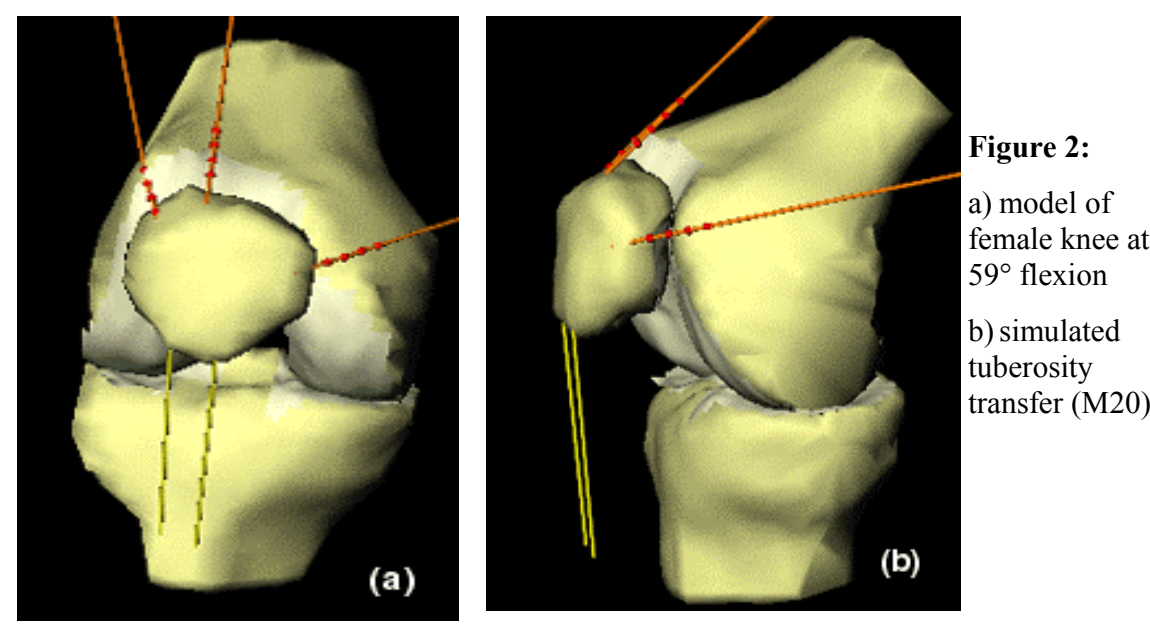

The tuberosity transfers were simulated by changing three inputs to the model. First, the force on the VL was decrease to $75 \%$ of its original value to simulate the lateral retinacular release, which typically accompanies a tuberosity transfer. Second, the insertion points on the tibia of the two line elements, which model the patellar ligament, were moved in the anterior or antero-medial directions (see Fig 2). The anatomical directions of the tibia were determined for each model using anatomic features, as described in our earlier work [15]. Finally, to maintain a constant flexion moment about the knee, the quadriceps forces were decreased to properly account for the increased moment arm of the patellar ligament resulting from the tuberosity transfer. The flexion moment prescribed to the model was derived from the peak closed-chain moment for rising from a chair, $82.2 \mathrm{~N} \cdot \mathrm{m}$ for males and $59.4 \mathrm{~N} \cdot \mathrm{m}$ for females [2]. For each simulation, including the initial pre-surgery simulation, the quadriceps forces were iteratively adjusted, keeping the ratio among them constant, until the model produced the desired flexion moment.

\subsection{Outcome Evaluation}

Several biomechanical variables were available for analyzing the results of the simulated surgery. Most importantly here, the model output contour maps showing the distribution of articular contact stress across the femur and patella cartilage surfaces; implicit in the use of this variable is the assumption that elevated articular stresses cause not only cartilage wear, but also joint pain. Clinically, the explicit motivation for the various PFJ tuberosity transfer procedures is indeed to reduce the magnitude of articular contact stress. For statistical comparison analyses, the mean stress across the surface and the peak stress were employed. The model yielded other useful data including: kinematics of the patella relative to the femur, articular contact area size, contact force, moment arm of the patellar ligament about the knee's helical (flexion) axis, and medial-lateral position of the centroid of the contact area.

In the analysis of results, the variables of mean articular contact stress and peak contact stress were normalized by their pre-surgery values for the purpose of statistical comparison across different patients. The flexion angles at which patients were imaged were not necessarily the same and, thus, the applied closed-chain flexion 
moment varied. An ANOVA with repeated measures was performed to compare the pre-surgical and post-surgical outcomes. A pairwise comparison was performed on the least squares (LS) means to detect differences between the various surgeries.
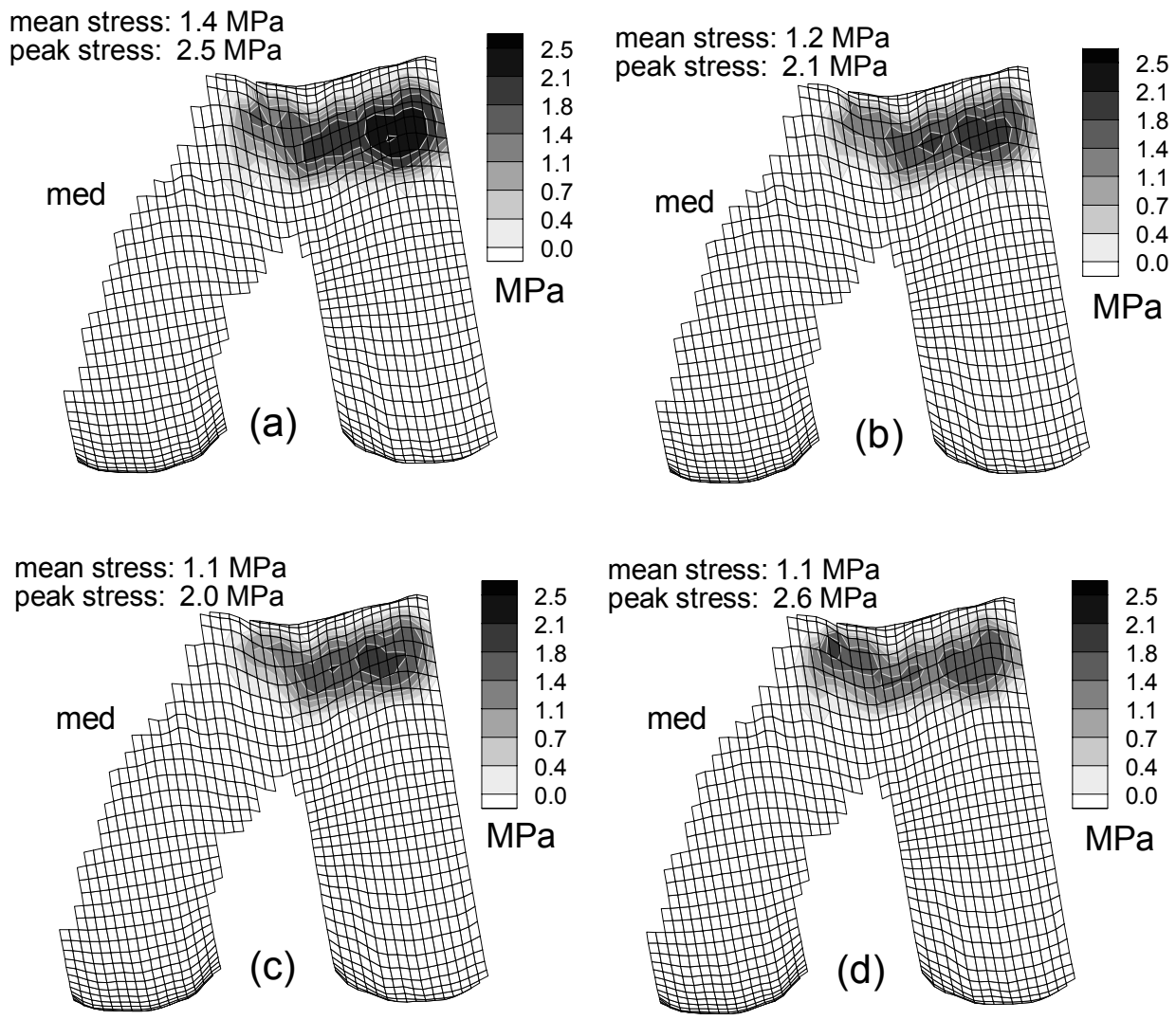

Figure 3: Patellofemoral contact stress maps for female patient with knee at $53^{\circ}$ flexion, displayed on femoral surface: a) pre-surgery; b) M15; c) M20; and d) F15-8.

\section{Results}

The articular contact stress pattern for a typical knee prior to surgery is presented in Figure 3a, where the model simulates a weak VMO and tight lateral retinaculum. This female patient was flexed at $53^{\circ}$, requiring an applied flexion moment of $31 \mathrm{~N} \cdot \mathrm{m}$. In this pre-surgical configuration, the mean stress is $1.36 \mathrm{MPa}$ and peak stress $2.53 \mathrm{MPa}$. Following M15 and M20 anteriorization procedures, the stress patterns were altered as demonstrated in Figures $3 \mathrm{~b}$ and $3 \mathrm{c}$. The mean stress decreased by $11 \%$, to $1.20 \mathrm{MPa}$, and by $16 \%$, to $1.14 \mathrm{MPa}$, for the two procedures respectively. The centroid of contact shifted only $0.1 \mathrm{~mm}$ medially for M15 and $0.8 \mathrm{~mm}$ laterally for M20. The impact of an $8 \mathrm{~mm}$ medial shift of the tuberosity is demonstrated in Figure $3 \mathrm{~d}$. The mean stress decreased by a total of $18 \%$ to $1.11 \mathrm{MPa}$ when the medialization was combined with the $15 \mathrm{~mm}$ anteriorization, while the contact area centroid shifted by $2.7 \mathrm{~mm}$ medially relative to the pre-surgical position, yielding a more uniformly 
distributed contact pattern. It can be noted that for this patient the F15-8 procedure produced the greatest decrease in stress and most centralized contact pattern, making it the best candidate procedure based on biomechanical criteria.
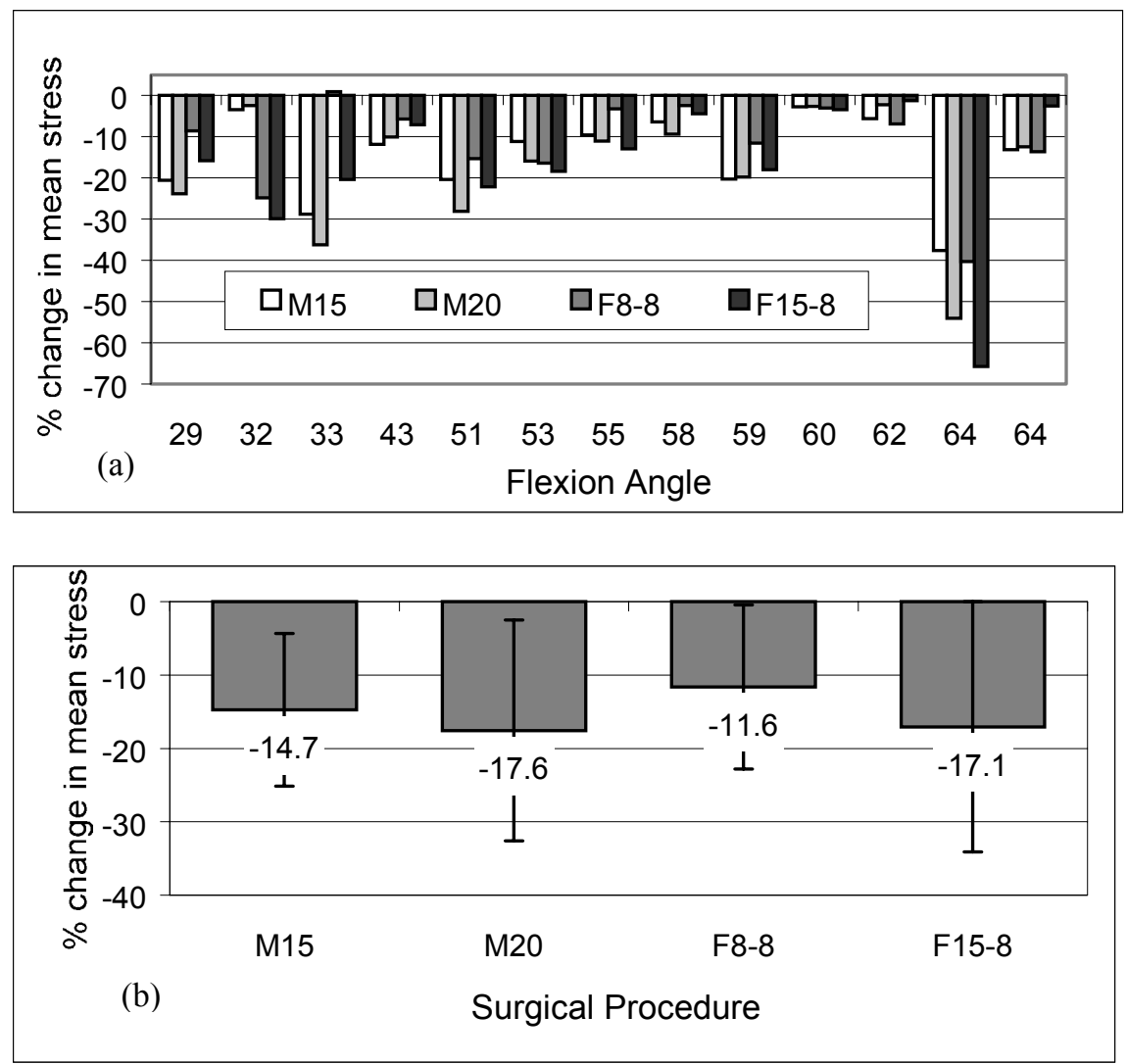

Figure 4: (a) Change in mean articular contact stress as a percentage of pre-surgery values for all 13 patients. The numbers on the horizontal axis represent the flexion angles at which the analysis was performed. (b) Average change in mean stress for $n=13$ from pre-surgery, $\mathrm{p}<0.0001$

When looking at the results for all patients however, results are more variable (Figures $4,5,6)$. On average, surgery produced a statistically significant $(\mathrm{p}=0.004)$ decrease in mean contact stress relative to pre-surgical conditions (Figure 4b), though a Duncan's multiple range test demonstrated no difference among the various surgical procedures. When examining the patient-specific results (Figure 4a), only six of thirteen knees achieved a decrease of more than $20 \%$ of the original stress for any of the procedures, while three knees demonstrated no better than a 10\% decrease. M15 achieved the best result in 2 patients, M20 in 4 patients, F8-8 in 2 patients, and F8-15 in 5 patients, thus emphasizing the variability among patients. When examining changes in peak articular contact stress relative to pre-surgical conditions (Figure 5), it is also found that, on average, surgery reduced the peak contact stress significantly 
$(\mathrm{p}=0.014)$, with Duncan's multiple range test indicating a grouping of pre-surgery and F8-8 against a grouping of M20, M15, and F15-8. However, patient-specific results indicate that certain surgical procedures may actually increase peak stresses in some subjects, as demonstrated in four of 13 patients. Importantly, increasing the amount of tuberosity transfer, e.g., from M15 to M20 or from F8-8 to F15-8 did not necessarily lead to a decrease in mean or peak stress, emphasizing that the patientspecific articular surface topography strongly influences the potential success of these surgical procedures.
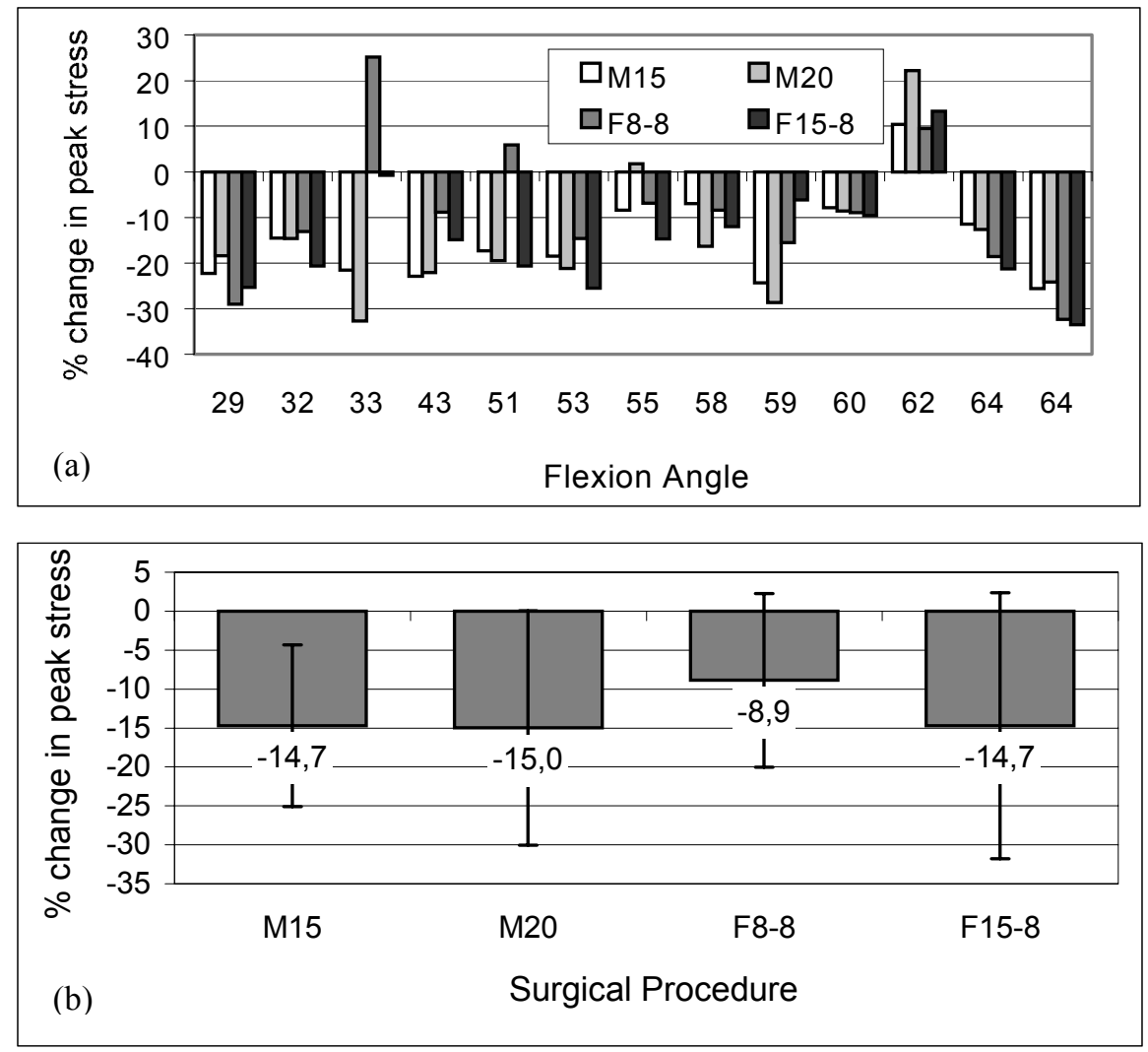

Figure 5: (a) Change in peak contact stress as a percentage of pre-surgery stress; (b) Average change in peak stress for $n=13$ from pre-surgery value, $\mathrm{p}<0.001$

Another outcome measure of the simulated surgical procedures is the amount by which the centroid of the contact area shifted medially (Figure 6). Not surprisingly, the Fulkerson procedure (F8-8 or F15-8) produced a consistent and statistically significant medial shift of the contact area relative to pre-surgical conditions $(\mathrm{p}<.0001)$; however, the outcome of the Maquet procedure (M15 or M20) is less consistent, showing no difference relative to pre-surgery according to Duncan's multiple range test $(\mathrm{p}=0.227$ and $\mathrm{p}=0.439)$. 

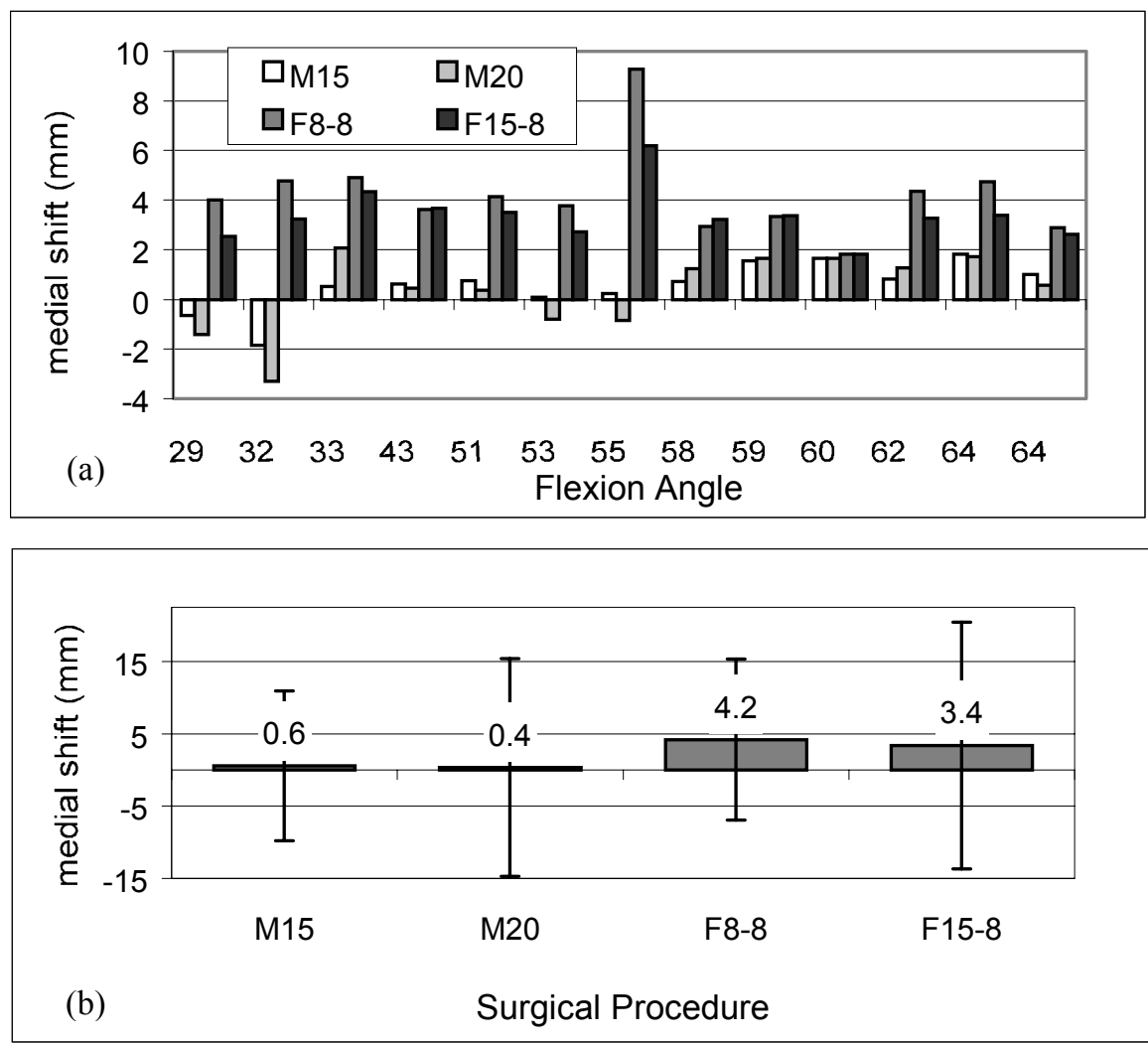

Figure 6: (a) Medial shift of stress centroid for 13 patients, 4 procedures; (b) Average shift for $\mathrm{n}=13$ from pre-surgery location, $\mathrm{p}<0.0001$

\section{Discussion}

For the 13 patients in this study the mean stress across the articular surfaces of the PFJ decreased following surgical simulation, although the magnitude of decrease varied considerably among patients. For example, comparing M20 to M15, one patient sees the mean stress decrease by an additional $17 \%$ while another sees the stress increase by 3\%. Although neither the Maquet nor the Fulkerson procedure stands out as universally superior to the other, for a given patient it is possible to identify one procedure as being superior to the other. While F15-8 was the superior procedure (as measured by decrease in mean stress) for the highest number of patients, i.e. 5 of 13, M20 was a close second demonstrating superiority for 4 of 13 patients.

This model accounted for individual surface topography by using in vivo data to describe the articular cartilage layer and by calculating articular contact stress based on local cartilage thickness. Since the size and location of lesions as well as the general surface topography are not consistent among patients, it is not surprising that the biomechanical outcomes of the surgical simulations produced results that varied from patient to patient. 
The results of this study are consistent with clinical findings that suggest that not all patients respond equally well to a given reconstructive procedure of the PFJ. It remains to ascertain whether some of the biomechanical variables employed provide a better predictive outcome of surgery than do others. For example, it is not known whether peak articular contact stress correlates better with joint pain than does mean contact stress. Such studies can be performed by following up on those patients who have undergone surgery and comparing their clinical outcome to the predictions of the model.

All simulations were performed for one flexion angle per patient. To fully predict the outcome of PFJ reconstructive surgery, analyses would have to be performed at more flexion angles. With the advent of open-MRI systems and high-resolution surface coils it is now possible to image a patient through a range of motion. Using the principles presented here, such imaging data could be used to predict the outcome of surgery for a more complete range of motion.

\section{Conclusion}

This study demonstrates that it is possible to perform cogent surgical simulations of corrective PFJ surgery. The analyses in this study were performed on PFJ models acquired from symptomatic patients, as opposed to cadavers with an unknown clinical history. Using the simulations it was possible to establish the best procedure for each individual patient. The results of this study are consistent with clinical findings of outcomes that differ from patient to patient. Further clinical studies are required to determine whether these surgical predictions in fact correlate with objective and subjective surgical outcomes.

\section{Acknowledgement}

We thank Mr. Peter Kung and Mr. Bryte Moa-Anderson for their help in data processing. We are grateful to The Whitaker Foundation for graduate student support.

\section{References:}

1. Ahmed, A.M., Burke, D.L., Yu, A. In-vitro measurement of static pressure distribution in synovial joints--Part II: Retropatellar surface. J Biomech Eng 105 (1983) 226-36.

2. Andriacchi, T.P., Natarajan, R.N., Hurwitz, D.E. Musculoskeletal Dynamics, Locomotion, and Clinical Applications. In: Mow, V.C. and Hayes, W.C. (eds.): Basic Orthopaedic Biomechanics, Raven Press, New York, (1991) 51 -92.

3. Ateshian, G.A., Kwak, S.D., Soslowsky, L.J., Mow, V.C. A stereophotogrammetric method for determining in situ contact areas in diarthrodial joints, and a comparison with other methods. J Biomech 27 (1994) 111-24.

4. Benvenuti, J.F., Rakotomanana, L., Leyvraz, P.F., Pioletti, D.P., Heegaard, J.H., Genton, M.G. Displacements of the tibial tuberosity. Effects of the surgical parameters. Clin Orthop (1997) 224-34.

5. Cohen, Z.A., Ateshian, G.A. The Effect of Cartilage Layer Thickness on Patellofemoral Joint Kinematics and Contact Stresses. ASME Adv in Bioeng BED (1999)

6. Cohen, Z.A., Kwak, S.D., Ateshian, G.A., Blankevoort, L., Henry, J.H., Grelsamer, R.P. , et al. The effects of tibial tuberosity transfer on the patellofemoral joint: A 3-D simulation. ASME Adv in Bioeng BED 33 (1996) 387-8. 
7. Cohen, Z.A., McCarthy, D.M., Kwak, S.D., Legrand, P., Fogarasi, F., Ciaccio, E.J. , et al. Knee cartilage topography, thickness, and contact areas from MRI: in-vitro calibration and in-vivo measurements. Osteoarthritis Cartilage 7 (1999) 95-109.

8. Cohen, Z.A., McCarthy, D.M., Roglic, H., Henry, J.H., Rodkey, W.G., Steadman, J.R. et al. Computer-Aided Planning of Patellofemoral Joint OA Surgery: Developing Physical Models from Patient MRI.. In: Wells WM, C.A., Delp S (eds.): Medical Image Computing and Computer Assisted Intervention, Springer-Verlag, (1998) 9-20.

9. Farahmand, F., Senavongse, W., Amis, A.A. Quantitative study of the quadriceps muscles and trochlear groove geometry related to instability of the patellofemoral joint. J Orthop Res 16 (1998) 136-43.

10. Ferguson AB, J.r., Brown, T.D., Fu, F.H., Rutkowski, R. Relief of patellofemoral contact stress by anterior displacement of the tibial tubercle. J Bone Joint Surg [Am] 61 (1979) 159-66.

11. Ferrandez, L., Usabiaga, J., Yubero, J., Sagarra, J., de No, L. An experimental study of the redistribution of patellofemoral pressures by the anterior displacement of the anterior tuberosity of the tibia. Clin Orthop (1989) 183-9.

12. Fulkerson, J.P., Becker, G.J., Meaney, J.A., Miranda, M., Folcik, M.A. Anteromedial tibial tubercle transfer without bone graft. Am J Sports Med 18 (1991) 490-6; discussion 496-7.

13. Heatley, F.W., Allen, P.R., Patrick, J.H. Tibial tubercle advancement for anterior knee pain. A temporary or permanent solution. Clin Orthop 208 (1986) 215-24.

14. Hirokawa, S. Three-dimensional mathematical model analysis of the patellofemoral joint. J Biomech 24 (1991) 659-71.

15. Kwak, S.D., Ahmad, C.S., Gardner, T.R., Grelsamer, R.P., Henry, J.H., Blankevoort, L. , et al. Hamstrings and iliotibial band forces affect knee kinematics and contact pattern. J Orthop Res 18 (2000) 101-8.

16. Kwak, S.D., Blankevoort, L., Ateshian, G.A. A 3D quasi-static mathematical multibody model for diarthrodial joints. Computer Methods in Biomechanics and Biomedical Engineering (October 2000)

17. Lewallen, D.G., Riegger, C.L., Myers, E.R., Hayes, W.C. Effects of retinacular release and tibial tubercle elevation in patellofemoral degenerative joint disease. J Orthop Res 8 (1990) 856-62.

18. Maquet, P. Advancement of the tibial tuberosity. Clin Orthop (1976) 225-30.

19. Noll, B.J., Ben-Itzhak, I., Rossouw, P. Modified technique for tibial tubercle elevation with realignment for patellofemoral pain. A preliminary report. Clin Orthop 234 (1988) 178-82.

20. Roglic, H., Ateshian, G.A., Cohen, Z.A., Kwak, S.D., Gardner, T., Henry, J. , et al. A Computer Simulation of Tibial Tuberosity Elevation and Patellar Tendon Adhesions. Trans ORS 23 (1998) 617.

21. Schmid, F. The Maquet procedure in the treatment of patellofemoral osteoarthrosis. Long-term results. Clin Orthop (1993) 254-8. 